Revista de Biología Marina y Oceanografía

Vol. 48, №3: 601-611, diciembre 2013

DOI 10.4067/S0718-19572013000300016

Artículo

\title{
Ciclo reproductivo, longitud y edad de madurez de jurel Trachurus murphyi, en la costa de Chile
}

\author{
Reproductive cycle, length and age at maturity of jack mackerel \\ Trachurus murphyi, in the Chilean coast
}

\author{
Elson Leal ${ }^{1}$, Eduardo Díaz ${ }^{1}$, J.C. Saavedra-Nievas ${ }^{1}$ y Gabriel Claramunt ${ }^{2}$
}

\begin{abstract}
'División de Investigación Pesquera, Instituto de Fomento Pesquero, Blanco 839, Valparaíso, Chile. elson.leal@ifop.cl
\end{abstract} ${ }^{2}$ Departamento de Ciencias del Mar, Universidad Arturo Prat, Iquique, Chile

\begin{abstract}
The spawning period, length and age at maturity of jack mackerel (Trachurus murphyi) in the Chilean coast is analyzed. The histological gonadal inspection confirmed that reproductive period of this species ranges between September and January. During the spawning season of 2011 the length at maturity $\left(L_{50}\right)$ of females was estimated in $22.7 \mathrm{~cm}$ fork length (FL) equivalent to an age of 2.44 years $\left(E_{50}\right)$. For males, the limited presence of immature individuals, did not allow to estimate the $L_{50}$ and $E_{50}$. However, the observed data suggest that the maturation process is different between sexes. Males reached full maturity between 21 and $23 \mathrm{~cm} \mathrm{FL}$, around 2-3 years, while females did so between 24 and $25 \mathrm{~cm} \mathrm{FL}$, at 4 years. The length at maturity in females did not show significant differences from previous estimates conducted through histological analysis. The lack of reproductive information in the beginning of the fisheries along with the differences in estimation methods, make difficult to reach reliable conclusions on a possible reduction in maturity length in females in recent years.
\end{abstract}

Key words: Trachurus murphyi, maturity, spawning, Chile

\begin{abstract}
Resumen.- Se analiza la época principal de desove y el proceso de maduración en longitud y edad del jurel (Trachurus murphyi) en la costa de Chile. El análisis histológico de las gónadas, confirmó que la actividad reproductiva de la especie tiende a concentrarse entre septiembre y enero. La longitud media madurez $\left(L_{50}\right)$ de las hembras durante la estación reproductiva del 2011 , se estimó en $22,7 \mathrm{~cm}$ de longitud de horquilla $(\mathrm{LH})$, equivalente a 2,44 años $\left(E_{50}\right)$. En el caso de los machos, la escasa presencia de ejemplares inmaduros, no permitió estimar satisfactoriamente $l_{a} L_{50}$ y $E_{50}$. Sin embargo, los datos observados sugieren diferencias importantes en el proceso de maduración entre sexos. Los machos alcanzaron la plena madurez sexual entre 21 y $23 \mathrm{~cm} \mathrm{LH}$, alrededor de los 2-3 años de vida, mientras que las hembras lo hicieron entre los 24 y $25 \mathrm{~cm} \mathrm{LH}$, a los 4 años. La $L_{50}$ de las hembras no varió significativamente de las estimaciones previas llevadas a cabo a través de análisis histológicos. Los escasos antecedentes reproductivos al comienzo de la pesquería, sumado a las diferencias en los métodos de estimación, impiden obtener conclusiones definitivas sobre una posible reducción en la longitud de madurez de las hembras durante los años recientes.
\end{abstract}

Palabras clave: Trachurus murphyi, madurez, desove, Chile

\section{INTRODUCCIÓN}

Un rasgo importante en la historia de vida de los peces teleósteos es la transición de la fase juvenil a la madurez sexual. En términos pesqueros y de evaluación de stock, la talla y/o la edad en que se alcanza la madurez sexual constituye un parámetro reproductivo básico ya que permite separar la fracción madura de un stock de peces. Se considera un componente crítico de la dinámica de poblaciones por su influencia en la tasa intrínseca de crecimiento poblacional. A menudo es usado como un punto biológico de referencia en un esfuerzo por permitir que los peces puedan reproducirse al menos una vez antes de ser capturados (Lowerre-Barbieri et al. 2011a).
El procedimiento de estimación de este parámetro reproductivo se basa en determinar ojivas de madurez en función de la talla o edad, sobre la base de un muestreo que cubra la mayor parte del área de distribución de la especie, con un adecuado tamaño de muestras. Es fundamental, que el muestreo también abarque el espectro de longitudes y/o edades de la población, especialmente en el rango de transición entre individuos inmaduros y maduros (Murua et al. 2003, Brown-Peterson et al. 2011), en una adecuada escala temporal (Hunter \& Macewicz 2003, Lowerre-Barbieri et al. 2011b). Asimismo, para una correcta descripción del proceso de maduración, se 
requiere de precisión en la categorización de los distintos estadios de desarrollo gonadal.

Las estimaciones de la longitud media de madurez $\left(\mathrm{L}_{50}\right)$ disponibles para el jurel (Trachurus murphyi) en el Pacífico Sur Oriental presentan un amplio rango que va de los 21,6 cm (Alegría et al. 1995) a los 39,0 cm de longitud horquilla (LH) (Andrianov 1985). Estas estimaciones se han realizado para diferentes áreas y períodos en las costas de Chile y Perú, considerado además diferentes metodologías y criterios de clasificación de los estadios de desarrollo gonadal. Sin embargo, los resultados de los análisis basados exclusivamente en la histología del tejido ovárico, reportan valores menos variables, con un promedio alrededor de los $23 \mathrm{~cm}$, un mínimo de 21,6 y un máximo de 26,7 cm LH (Cubillos \& Alarcón 2010). A pesar que la aproximación histológica es considerada más precisa y menos sesgada (Kjesbu et al. 2003, Murua \& Saborido-Rey 2003, Tomkiewicz et al. 2003) se pueden estimar diferentes ojivas de madurez con un mismo set de muestras, dependiendo de los criterios con los cuales los individuos son o no asignados dentro de la proporción madura de la población (Brown-Peterson et al. 2011, Lowerre-Barbieri et al. 2011a).

En la actualidad, el jurel no cuenta con una estimación de la talla media de madurez sexual con cobertura espacial a lo largo de toda la costa de Chile y bajo criterios de clasificación consensuados de los estadios de desarrollo gonadal. Asimismo, se desconocen posibles diferencias en el proceso de maduración en función de la longitud, entre machos y hembras. Tampoco se ha estudiado este proceso en relación con la edad basado en la lectura directa de los otolitos. De esta manera, el objetivo principal de este estudio fue analizar el ciclo reproductivo de $T$. murphyi y estimar una ojiva de madurez actualizada, sobre la base del análisis histológico de las gónadas.

\section{Materiales Y MÉTOdos}

El área de estudio comprendió la zona costera de Chile, entre los $18^{\circ} 21^{\prime}$ y $46^{\circ} 00^{\prime} S$ (Fig. 1). La información para desarrollar el estudio provino del monitoreo biológico de la especie, realizado por el Instituto de Fomento Pesquero de Chile (IFOP) cubriendo el área operación de la flota cerquera que captura el recurso en aguas nacionales. El diseño de muestreo correspondió al tipo estratificado simple, realizado mensualmente entre febrero 2011 y enero 2012. Complementariamente, para el estudio del ciclo reproductivo se utilizó información recopilada por IFOP entre enero 2006 y diciembre 2010. Se analizó la variación mensual del Índice Gonadosomático (IGS) y estados macroscópicos de madurez de las hembras, contabilizando un total de 40.822 registros. Las hembras fueron clasificadas dentro de 5 estadios de desarrollo de acuerdo a la escala de madurez de Holden \& Raitt (1975). El IGS se estimó a partir de una modificación de Nikolsky (1963), según la ecuación:

$$
\text { IĜS } S_{h}=\frac{\sum \mathrm{Wg}_{\mathrm{h}}}{\sum \mathrm{W}_{\mathrm{h}}} 100
$$

donde, IĜS $\mathrm{h}_{\mathrm{h}}$ es el promedio dentro de cada mes (h), $\mathrm{W}$, es el peso del ovario $(\mathrm{g}), \mathrm{W}_{\mathrm{h}}$ corresponde al peso eviscerado de las hembras.

Entre febrero 2011 y enero 2012, la actividad reproductiva, se describió sobre la base de la variación mensual del IGS y de la frecuencia de ejemplares maduros

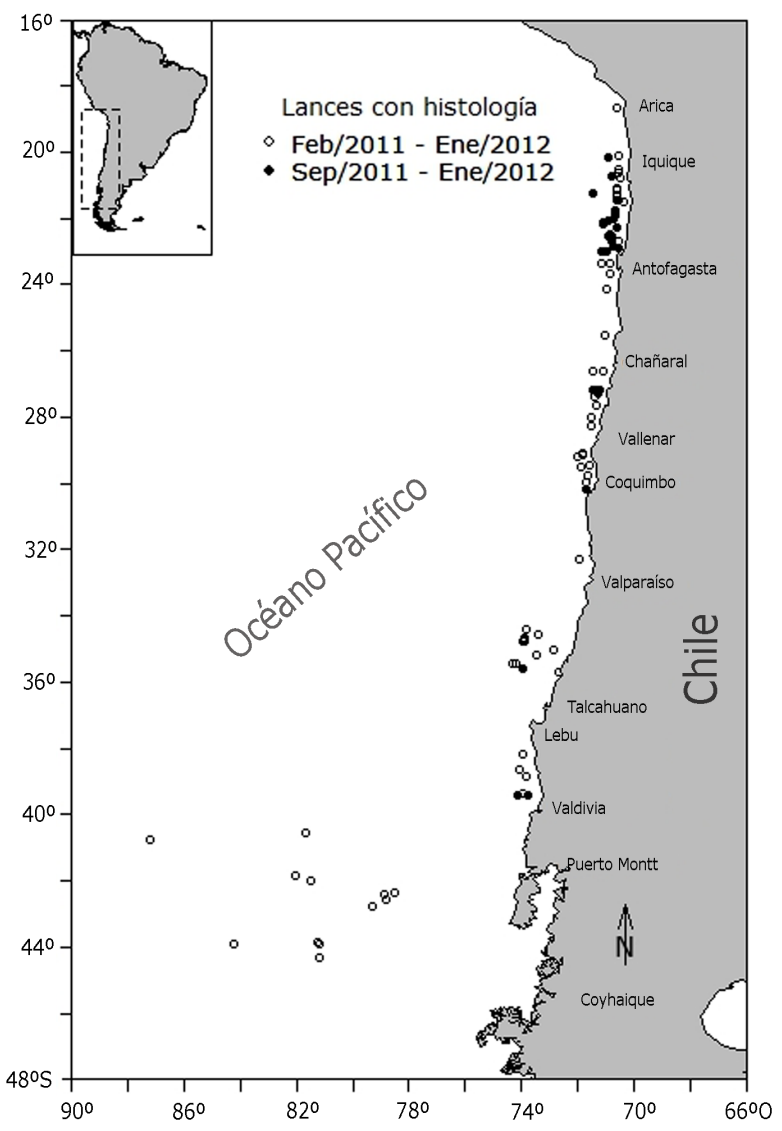

Figura 1. Área de estudio. Distribución de los lances de pesca para el monitoreo de la actividad reproductiva (febrero 2011-enero 2012) y estimación de la ojiva de madurez (septiembre 2011-enero 2012) / Study area. Distributions of fishing cast for monitoring to reproductive activity (February 2011-January 2012) and maturity ogive estimation (September 2011-January 2012) 
según la escala de desarrollo microscópica propuesta por Brown-Peterson et al. (2011) para machos y hembras peces desovadores parciales. Esta escala fue analizada y discutida en el marco de un taller internacional de revisión de criterios histológicos para la asignación de fases de madurez gonadal de jurel (Saborido-Rey \& Leal 2011) ${ }^{1}$. Junto a la descripción de cada fase de desarrollo gonadal, se presentan láminas representativas con las características cito-histológicas de hembras y machos de la especie (Tablas 1 y 2).

En terreno, cada pez fue medido, registrando su peso total (PT, $\pm 0,1 \mathrm{~g}$ ) y longitud de horquilla $(\mathrm{LH}, \pm 0,5 \mathrm{~cm})$. El testículo u ovario de cada ejemplar fue extraído, pesados $( \pm 0,1 \mathrm{~g})$ y fijados en formalina neutralizada y diluida al $10 \%$ para su posterior procesamiento. Se recopilaron durante el periodo de muestreo un total de 1.096 machos y 1.245 hembras.

En laboratorio, las muestras fueron procesadas histológicamente para asignar las diferentes fases de madurez. Para la obtención de los cortes histológicos, se utilizó la técnica recomendada por López et al. (1982). Las muestras fueron deshidratadas en alcohol, incluidas en parafina, cortadas a un espesor de $5 \mu$ y finalmente coloreadas con Hematoxilina y Eosina.

Se utilizó para el estudio de madurez en longitud un total de 456 machos y 478 hembras obtenidas en el periodo de mayor actividad reproductiva (septiembre 2011- enero 2012).

Para el estudio de la edad madurez en ambos sexos, además del tejido gonadal, se extrajeron los otolitos de cada ejemplar. La edad, fue estimada a través de las bandas de crecimiento que se encuentran contenidas en esta estructura. La lectura se realizó directamente sobre el otolito entero, usando aceite como líquido aclarador, luz reflejada y un aumento de 10x. Se analizaron un total de 112 otolitos en machos y 146 en hembras, correspondientes a muestras obtenidas entre septiembre 2011 y enero 2012.
Un modelo logístico fue utilizado para describir la proporción de ejemplares maduros por clase de longitud (L) o edad (E).

La ecuación que define la forma logística de la curva, está descrita por:

$$
\mathrm{P}=\frac{1}{1+\exp \left(\beta_{0}-\beta_{1} * \mathrm{~L}, \mathrm{E}\right)}
$$

donde $\mathrm{P}$ es la proporción de ejemplares maduros a la longitud (L) o edad (E), y $\beta_{0}$ y $\beta_{1}$, son los parámetros que representan la posición y la pendiente de la curva respectivamente. Estos parámetros fueron obtenidos por Máxima Verosimilitud, asumiendo una distribución binomial (maduro/inmaduro) de la variable aleatoria. La función de estimación log-verosímil tuvo la forma:

$$
\mathrm{L}\left(\beta_{0}, \beta_{1}\right)=\sum \mathrm{k} \ln (\mathrm{P})+(1-\mathrm{h}) \ln (1-\mathrm{P})
$$

donde $\mathrm{k}$ indica la presencia o ausencia de individuos maduros, y $\mathrm{P}$ es la función logística previamente descrita. Tanto machos como hembras fueron catalogados como maduros a partir de la fase II de la escala microscópica (en desarrollo). Dicha fase, en el caso de las hembras, se caracteriza por la presencia de alvéolos corticales desarrollados en el citoplasma del ovocito (Tabla 1)

La longitud o edad media de madurez queda definida cuando la función logística alcanza el 50\% de probabilidad de observar individuos maduros a esa clase de longitud $\left(\mathrm{L}_{50}\right)$ o edad $\left(\mathrm{E}_{50}\right)$ y se obtiene como la razón entre los parámetros $\beta_{0} \mathrm{y} \beta_{1}$, i.e.,

$$
\mathrm{E}_{50}, \mathrm{~L}_{50}=\frac{\beta_{0}}{\beta_{1}}
$$

El intervalo de confianza para la longitud media de madurez, se obtuvo mediante un enfoque de re-muestreo tipo Monte Carlo de los parámetros $\beta_{0}$ y $\beta_{1}$ según lo sugerido por Roa et al. (1999) para este tipo de análisis.

${ }^{1}$ Saborido-Rey F \& E Leal. 2011. Revisión de los criterios histológicos para la asignación de estados de madurez en jurel. Reporte Técnico. IFOP-Subsecretaría de Pesca, Valparaíso, 22 pp. + Anexos. 
Tabla 1. Descripción de las fases de desarrollo ovocitario en el ciclo reproductivo de las hembras de $\mathrm{T}$. murphyi. $(\mathrm{E}=$ Envoltura ovárica, $\mathrm{LO}=$ Lamela ovárica, $\mathrm{AC}=$ Alveolos corticales, $\mathrm{CN}=$ Cromatina nucleolar, $\mathrm{PN}=$ Perinucleolar, $\mathrm{CP}=\mathrm{crecimiento}$ primario,

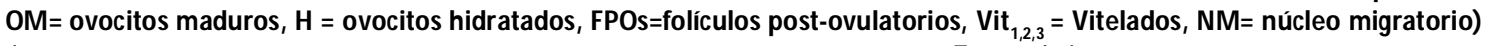
/ Description of the oocyte developmental phases in the reproductive cycle of T. murphyi. $(\mathrm{E}=$ Ovarian cover, $L O=$ Ovarian lamella, $\mathrm{AC}=$ Cortical alveolar, $\mathrm{CN}=$ Chromatin nucleolar, $\mathrm{PN}=$ Perinuclear, $\mathrm{CP}=$ Primary growth, $\mathrm{OM}=$ Oocyte maturation, $\mathrm{H}=$ Hydrated oocytes, $\mathrm{FPO}=\mathrm{s}=$ Postovulatory follicle, $\mathrm{Vit}_{1,2,3}=$ Vitellogenic, $\mathrm{NM}=$ Germinal vesicle migration)

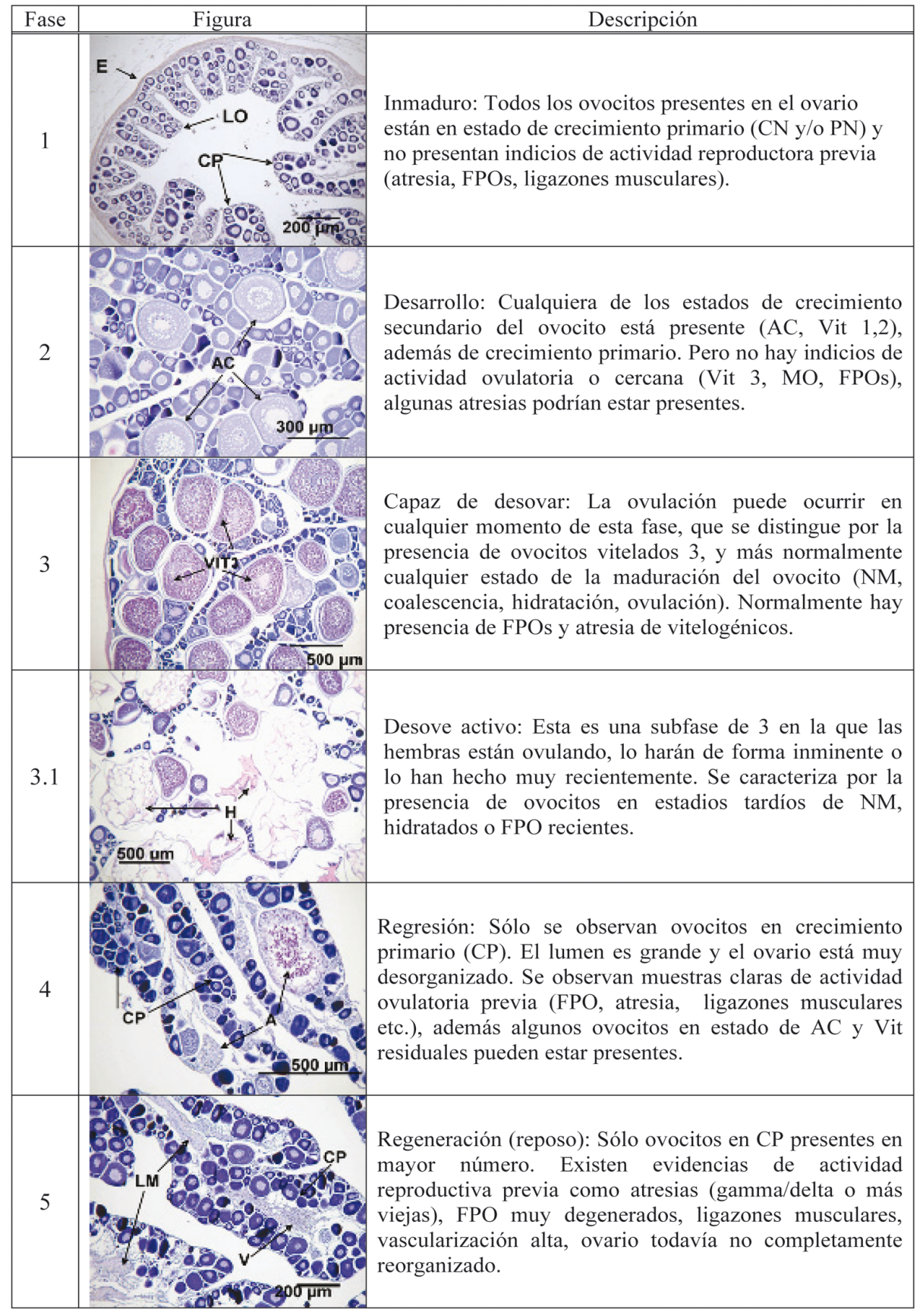


Tabla 2. Descripción de las fases la espermatogénesis en el ciclo reproductivo de T. murphyi. (NS = Nidos espermatogoniales, EG: Epitelio Germinal; SD = Espermatoductos, L = Lumen, LO = Lobulos, SP = Proliferación espermatogonial, Sc1: Espermatocito primario; Sc2: Espermatocito secundario; Sg1: Espermatogonia primaria; Sg2: Espermatogonia secundaria; St: Espermátida; Sz: Espermatozoide) / Spermatogenesis phases in the reproductive cycle of T. murphyi. (NS = Spermatogonial nest, EG = Germinal epithelium; SD = Spermatogonial duct, $\mathrm{L}=$ Lumen, $\mathrm{LO}=$ Lobe, $\mathrm{SP}=$ Spermatogonial proliferation, Sc1 = Primary spermatocyte; Sc2 = Secondary spermatocyte; Sg1 = Primary spermatogonia; Sg2 = Secondary spermatogonia; St = Spermatid; Sz = Spermatozoa)

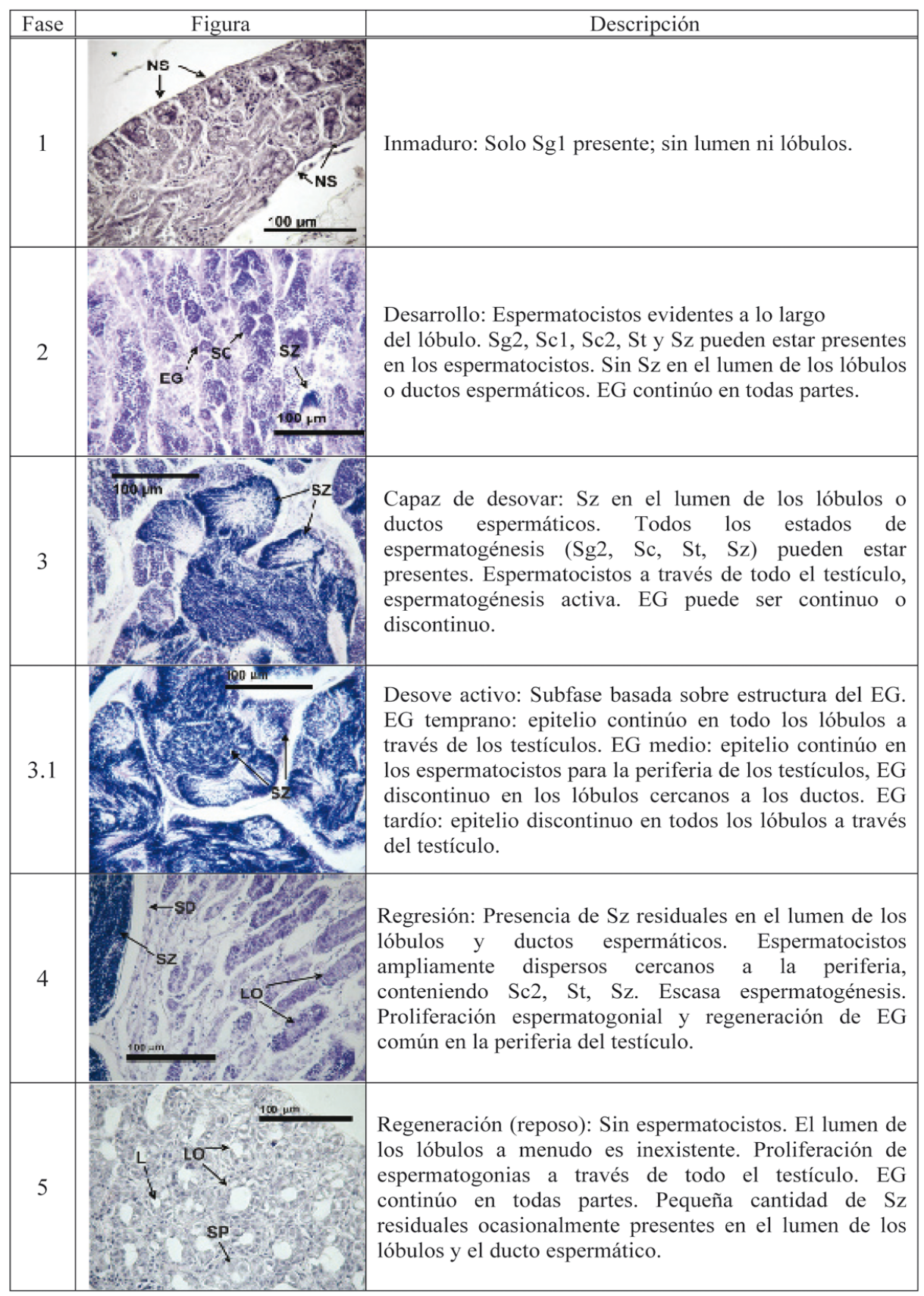


a)

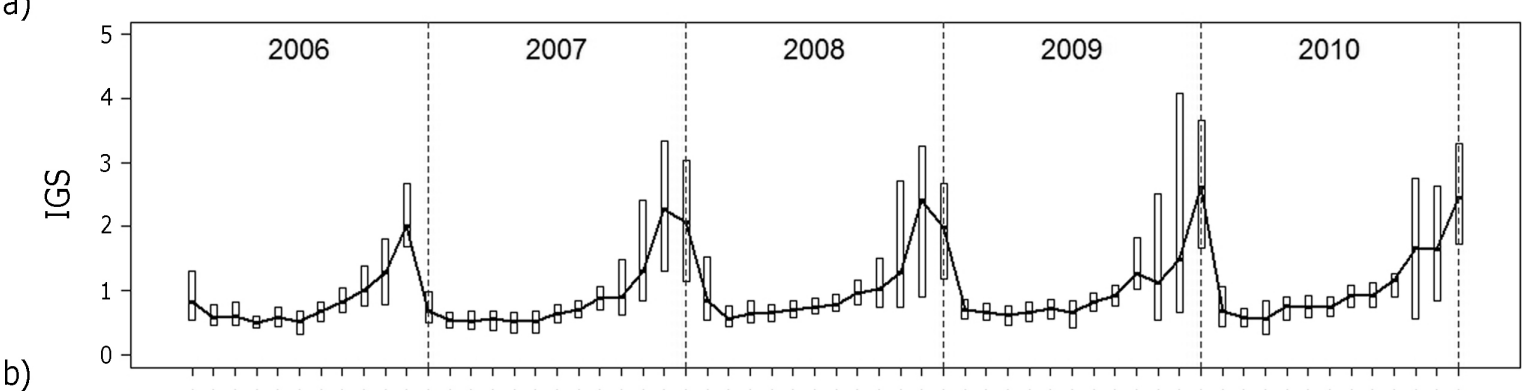

b)

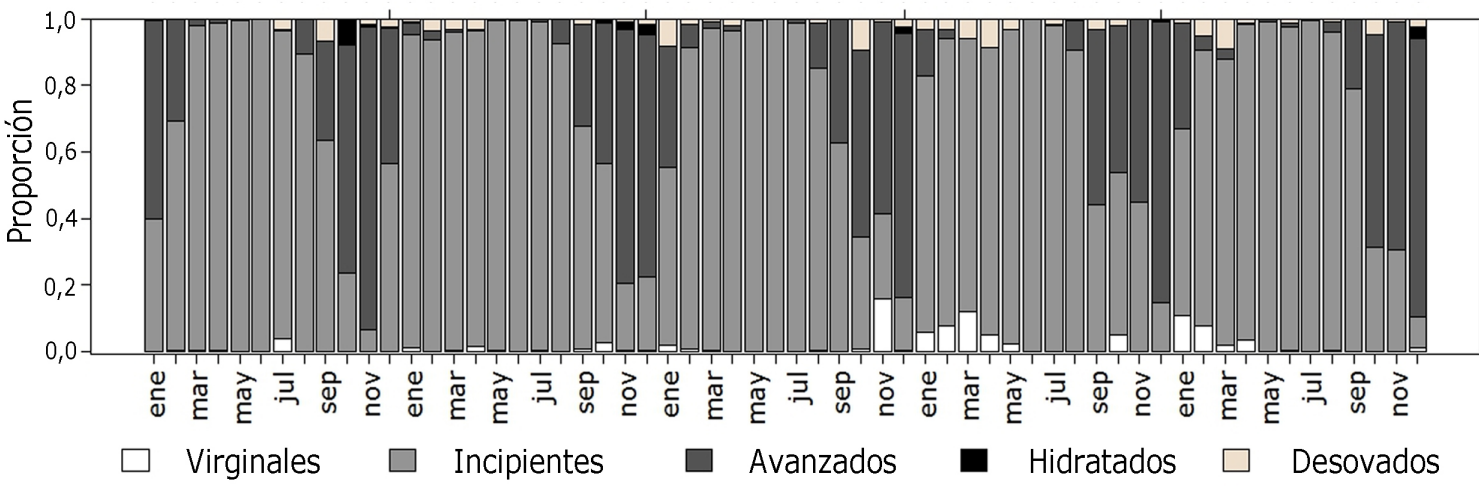

Figura 2. Variación mensual del IGS (a) y de la proporción de los estados de madurez macroscópicos (b) de las hembras de T. murphyi en la costa de Chile, entre el 2006 y 2010 / Monthly variation of the GSI (a) and of proportion of macroscopic maturity stages (b) of females of T. murphyi in the Chilean coast between 2006 and 2010

\section{Resultados}

\section{Ciclo reproductivo}

El análisis del IGS, así como la frecuencia de estados macroscópicamente maduros de las hembras analizadas entre el 2006 y 2010, revelaron un incremento de la actividad reproductiva de T. murphyi en el segundo semestre, durante la primavera y principios del verano austral (Fig. 2).

El IGS promedio mostró valores elevados entre los meses de octubre y diciembre de cada año, excepto en diciembre del 2006 (Fig. 2a). De manera similar, aunque se observó la presencia de ovarios con algún grado de madurez durante todo el año, el análisis macroscópico indicó una mayor frecuencia de hembras en estado de madurez avanzada entre agosto y diciembre. Por su parte, las hembras en evidente actividad reproductiva (ovarios hidratados), aunque fueron poco frecuentes, estuvieron siempre asociados al último trimestre del año (Fig. 2b).

Entre febrero 2011 y enero 2012, el ciclo reproductivo medido a través del IGS confirmó el patrón observado entre 2006 y 2010, excepto en noviembre, cuando el valor medio obtenido fue inferior al histórico (Fig. 3). El análisis

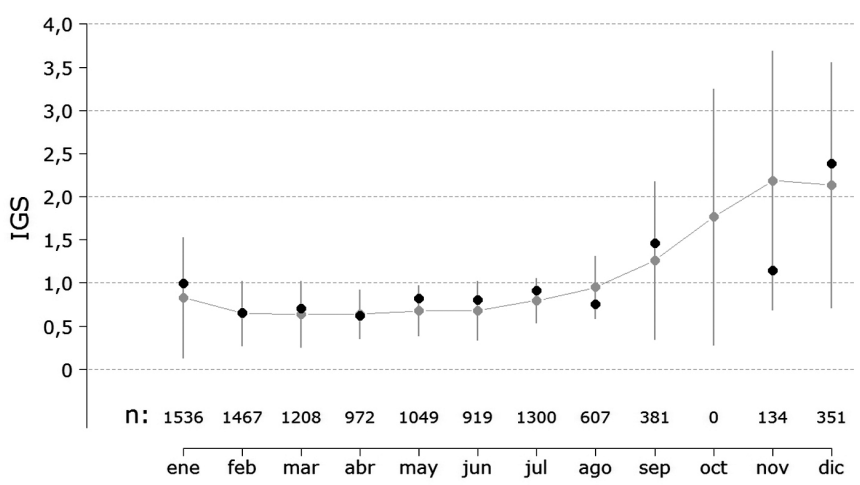

Figura 3. Patrón global del IGS entre el 2006 y 2010 y su desviación estándar (círculos y líneas grises) y su variación mensual durante el 2011 (ćŕrulos negros). Junto al eje $x$, número de registros usados en la estimación del índice durante el 2011. Las barras verticales corresponden a la desviación estandar / GSI global pattern between 2006 and 2010 (grey circles and lines) and GSI monthly variation in 2011 (black circles). On $x$-axis the number fish used in the index estimation during 2011 is indicated. Vertical bars denote standard deviation 


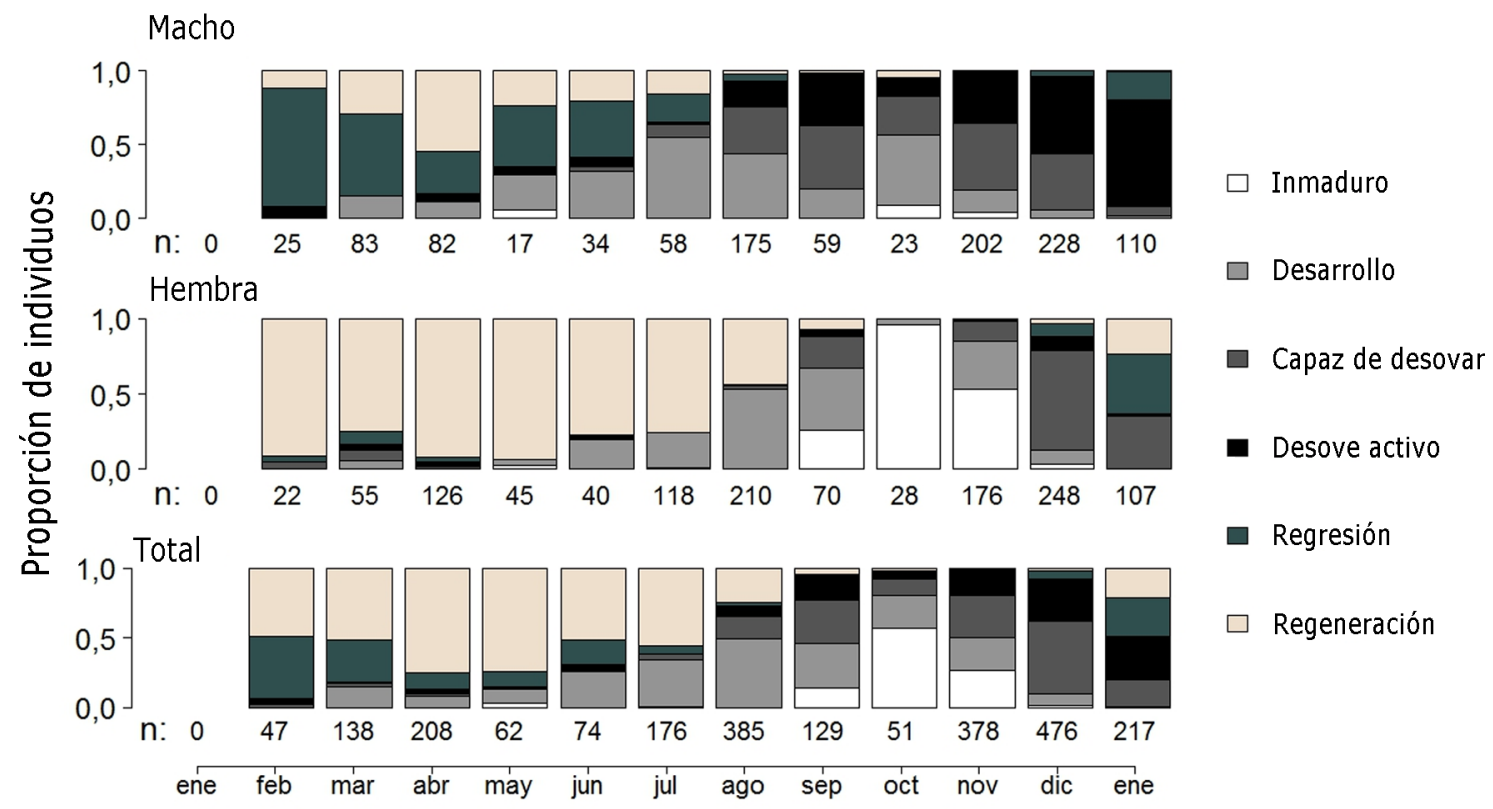

Figura 4. Proporción mensual de los estados de madurez (EM) microscópicos de machos, hembras y para el total de las muestras de T. murphyi en la costa de Chile entre febrero 2011 y enero 2012. Valor bajo las barras corresponde al número de muestras / Monthly proportion of microscopic maturity stages (EM) of males, females and for the overall samples of T. murphyi in the Chilean coast from February 2011 to January 2012. Value under bar denote sample number

histológico en tanto, corroboró que la actividad reproductiva de machos y hembras ocurre principalmente entre septiembre y enero (Fig. 4).

En el caso de los machos, el análisis histológico reveló una proporción importante de individuos en capacidad reproductiva y fase de desove activo a partir de agosto. En las hembras, esto se apreció a partir de septiembre, aunque en octubre se observaron mayoritariamente ejemplares inmaduros.

El análisis de la información en conjunto confirmó un periodo de reposo reproductivo entre febrero y julio, donde predominaron los ejemplares en regresión y regeneración.

\section{LONGITUD DE MADUREZ}

El rango de tamaños de las hembras analizadas para el estudio de madurez en longitud fluctuó entre los 20 y 39 cm LH. Los resultados del modelo logístico indican un rápido proceso de maduración entre los 20 y los $25 \mathrm{~cm} \mathrm{LH}$ (Fig. 5a). A los $21 \mathrm{~cm}$ LH sólo el 3\% de las hembras fue catalogada como madura, mientras que a los $24 \mathrm{~cm} \mathrm{LH}$ todas fueron asignadas dentro de la fracción madura de la población. La longitud media de madurez $\left(\mathrm{L}_{50}\right)$ de las hembras de T. murphyi entre septiembre 2011 y enero 2012, se estimó en 22,7 cm LH (Tabla 3).

Tabla 3. Longitud media de madurez $\left(L_{50}\right)$ de las hembras de $T$. murphyi, límite inferior $\left(L_{\text {inf }}\right)$ y superior $\left(L_{\text {sup }}\right)$ para $L_{50}$. Parámetros de la ecuación logística $\left(\beta_{0}\right.$ y $\left.\beta_{1}\right)$ y su error estándar en paréntesis / Mean of length at maturity $\left(L_{50}\right)$ for T. murphyi females, lower limit $\left(L_{\text {inf }}\right)$ and upper limit $\left(L_{\text {sup }}\right)$ to $L_{50}$. Logistics equation parameters $\left(\beta_{0}\right.$ and $\left.\beta_{1}\right)$ and standard error in parenthesis

\begin{tabular}{ccc}
\hline \multirow{2}{*}{ Parámetro } & \multicolumn{2}{c}{ Análisis } \\
\cline { 2 - 3 } & Longitud & Edad \\
\hline $\mathrm{L}_{\text {inf }}$ & 22,3 & 2,03 \\
$\mathrm{~L}_{50}$ & 22,7 & 2,45 \\
$\mathrm{~L}_{\text {sup }}$ & 23,2 & 2.98 \\
$\beta_{0}$ & $32,93(15,09)$ & $4,31(17,9)$ \\
$\beta_{1}$ & $1,45(0,67)$ & $1,76(5,08)$ \\
\hline
\end{tabular}



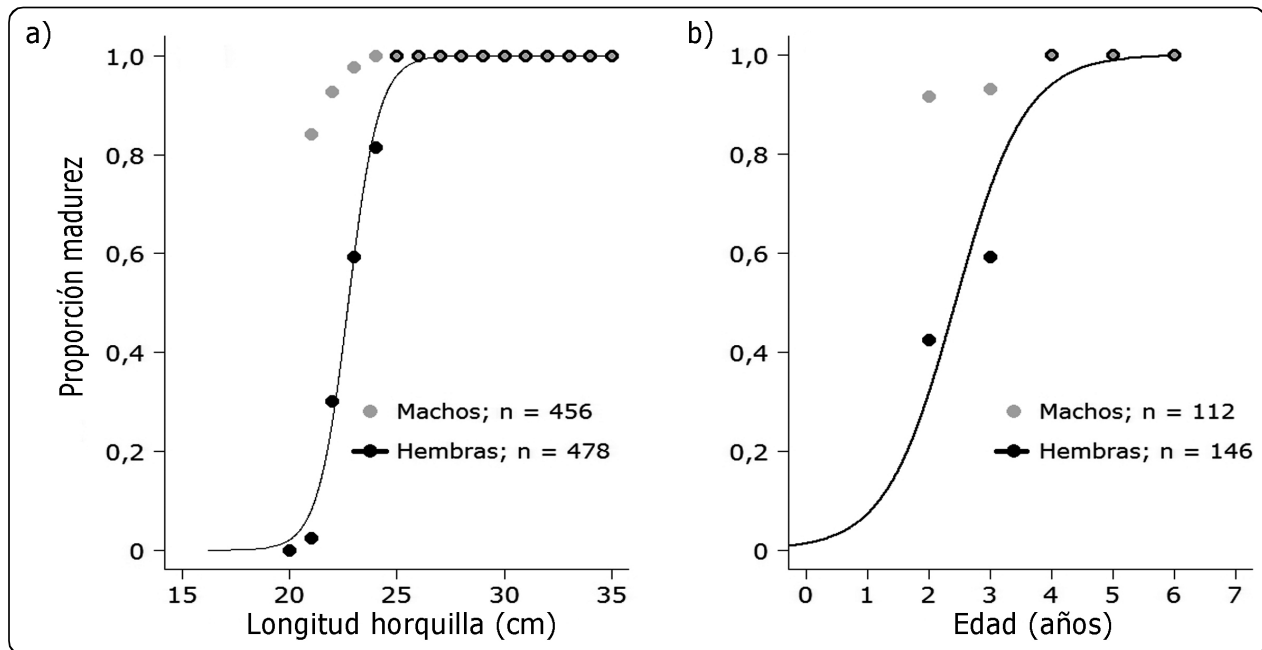

Figura 5. Ojiva de madurez en longitud (a) y edad (b) de machos y hembras de T. murphyi en la costa de Chile / Length (a) and age (b) maturity ogives of males and females of T. murphyi in the Chilean coast

En el caso de los machos, el rango de tamaños de los peces analizados varió entre 20 y $42 \mathrm{~cm}$ LH. Los ejemplares inmaduros tuvieron una escasa representatividad dentro de las muestras. De los 456 individuos analizados, sólo 10 (entre 21 y $23 \mathrm{~cm} \mathrm{LH}$ ) fueron catalogados como inmaduros. Por esta razón, debido a la falta de observaciones en la primera parte de la curva, no fue posible ajustar una ojiva de madurez satisfactoria para los machos. Sin embargo, a pesar de la ausencia de un ajuste, los datos observados indicaron que entre los $21 \mathrm{y}$ $23 \mathrm{~cm}$ LH el porcentaje de machos maduros alcanzó el $85 \%$ y $100 \%$ respectivamente (Fig. 5a).

\section{EDAD DE PRIMERA MADUREZ}

El rango de edad de las hembras analizadas fluctuó entre los 2 y 6 años. La edad media de madurez $\left(\mathrm{E}_{50}\right)$ se estimó en 2,4 años. Los resultados del modelo logístico indican que por debajo de los 2 años de edad la proporción de hembras maduras disminuye considerablemente, mientras que a partir de 4 años existe una alta probabilidad que la mayoría se encuentren maduras (Fig. 5b). El intervalo de confianza al $95 \%$ para la edad de madurez $\left(\mathrm{E}_{50}\right)$ de las hembras, así como los parámetros $\beta_{0}$ y $\beta_{1}$ que describen la ecuación logística, se resumen en la Tabla 3 .

En los machos, al igual que en las hembras, el rango de edad de los individuos analizados fluctuó entre los 2 y los 6 años. Similar a lo observado con la talla, la escasa proporción de ejemplares machos inmaduros en las muestras impidieron estimar un valor verosímil para la $\mathrm{E}_{50}$. Sin embargo, los datos observados también sugieren diferencias en el proceso de maduración entre sexos. Los resultados revelaron que la mayoría de los machos alcanzan la madurez sexual entre el primer y tercer año de vida, mientras que las hembras lo hacen entre el tercer y cuarto año (Fig. 5b).

\section{Discusión}

Los objetivos del presente estudio se fundamentaron en la necesidad de contar con una estimación actualizada de la longitud y edad de madurez de T. murphyi en las aguas chilenas, a partir de un análisis detallado del proceso de maduración en esta especie. Uno de los requisitos fue establecer además una escala de madurez basada en criterios consensuados, que pueda ser usada como referencia en futuros estudios reproductivos del jurel en la región.

Para disminuir la incertidumbre en el reconocimiento de un individuo inmaduro de uno maduro en reposo reproductivo (inactivo), es importante que la ojiva de madurez sea estimada en el período de mayor actividad reproductiva de la población. Por esta razón, se analizó primero el ciclo reproductivo anual de la especie a través de índices macroscópicos durante los cinco años previos al desarrollo del presente estudio. Los resultados de este análisis fueron comparados luego con el monitoreo histológico del tejido reproductivo de machos y hembras.

Los análisis de la actividad reproductiva demostraron que el periodo principal de desove de T. murphyi en la costa de Chile se extiende entre septiembre y enero. Tales resultados concuerdan con reportes previos que indican 
una época de mayor actividad reproductiva en los meses de primavera-verano con máximos entre octubre y noviembre (Arancibia \& Cubillos 1993, Grechina et al. 1998). Oyarzún et al. (1998) y Aracena et al. (1998) por su parte, señalan que la máxima actividad reproductiva de la especie se extiende hasta enero en aguas costeras y oceánicas frente a Chile. Las escasas hembras analizadas durante octubre, correspondieron a ejemplares pequeños inmaduros provenientes de la zona norte. Esto impidió corroborar el incremento en la actividad reproductiva observada en septiembre, donde el número de muestras y el rango de longitudes fueron mayores.

La $\mathrm{L}_{50}$ de las hembras estimada en $23 \mathrm{~cm} \mathrm{LH}$, correspondería según la lectura de otolitos a una edad de 2,4 años $\left(\mathrm{E}_{50}\right)$. Los machos por su parte, mostraron una baja proporción de ejemplares inmaduros, apareciendo escasamente en las muestras. La falta de observaciones en la sección inferior de las curvas de madurez en longitud y edad, impidió obtener ojivas robustas desde el punto de vista estadístico. A pesar de estas limitaciones, los datos observados sugieren diferencias importantes en el proceso de maduración en longitud y edad de machos y hembras. Entre los 21 y $23 \mathrm{~cm} \mathrm{LH} \mathrm{(2-3} \mathrm{años),} \mathrm{existe} \mathrm{una}$ alta probabilidad de encontrar el $100 \%$ de los machos maduros, mientras que en las hembras, esto se observa entre $\operatorname{los} 24$ y 25 cm LH (4 años).

Las diferencias en el proceso de maduración entre sexos son consistentes con lo reportado por Andrianov (1985), quien señala una madurez más temprana en machos que en hembras de T. murphyi en aguas costeras del Perú. Esta observación es coincidente con lo reportado para otros representantes de la familia, como es el caso de $T$. trachurus en el Atlántico norte (Abaunza et al. 2003).

Diferencias en los rasgos de historia de vida entre sexo son características comúnmente observada en distintas especies de peces, principalmente aquellas que son recursos pesqueros de importancia (Lucio et al. 2000, Piñeiro \& Saínza 2003). Las explicaciones en este sentido señalan posibles diferencias metabólicas, por ejemplo en el consumo del oxígeno y/o diferencias en el costo energético entre la reproducción y crecimiento entre sexos (Pauly 1994, Rijnsdorp \& Ibelings 1989).

La longitud y edad de madurez estimada para $T$. murphyi puede ser considerada precoz para una especie que puede alcanzar los $60 \mathrm{~cm} \mathrm{LH}$ (Kochkin 1994), con edades máximas estimadas de hasta 16 años (Abramov \& Kotlar 1980). Sin embargo, la $\mathrm{L}_{50}$ de las hembras estimada en el presente estudio, se encuentra dentro de los valores medios reportados previamente para la especie en diferentes periodos y áreas frente a la costa de Chile y Perú. Estos valores, resumidos por Cubillos \& Alarcón (2010), variaron principalmente entre los 21 y $25 \mathrm{~cm} \mathrm{LH}$, cuando fue usada la técnica histológica para asignar fases de madurez. Los resultados también concuerdan con la estimación en edad indicada por Kaiser (1973), quien señala que las hembras de T. murphyi frente a las costas de Chile podrían desovar por primera vez durante el tercer año de vida. Andrianov (1985) por su parte, reporta una madurez temprana de la especie entre el tercer y cuarto año de vida, en aguas del Perú. De acuerdo a Marshall et al. (1980), T. symmetricus en el hemisferio norte, podría madurar precozmente al segundo año de vida. De la misma forma, Abaunza et al. (2003), catalogan a T. trachurus como una especie longeva (hasta 40 años) que madura tempranamente entre el segundo y cuarto año de vida.

Una posible explicación para una temprana maduración de la especie, puede estar relacionada a la alta tasa de explotación a la que ha estado sometido el jurel durante las últimas décadas. Los estudios señalan que la longitud y edad de madurez de poblaciones sometidas a explotación intensa podrían disminuir (Rochet 2009), por lo que los parámetros de la historia de vida cambiarían en respuesta a la actividad pesquera (Fenberg \& Roy 2008, Matsumura et al. 2011). Estos cambios, serían el resultado de la plasticidad fenotípica, aunque en el largo plazo la presión selectiva de la pesca puede llevar a cambios a nivel genético (Araya \& Pepe-Victoriano 2010).

Según Conover \& Munch (2002), la selección de tamaños debido al arte de pesca, remueve preferentemente individuos de crecimiento rápido que reclutan a la pesquería a edades más jóvenes, dejando en el agua aquellos genotipos de crecimiento lento. Así la talla de madurez se reduce, aunque no necesariamente la edad. Estos genotipos comienzan a predominar y son transmitidos entre generaciones, afectado la tasa de crecimiento poblacional.

Los resultados de este estudio, son escasamente contrastables con estimaciones realizadas en periodos de explotación baja o moderada de la especie (década de los 70‘). El único antecedente disponible (Kaiser 1973) se basó en el color de los ovocitos y no en histología para la clasificación de madurez. El estudio, tampoco logró colectar los individuos más pequeños $(<34 \mathrm{~cm} \mathrm{LH})$. Los resultados coinciden con la edad de madurez estimada en el presente trabajo, pero difirieron significativamente en la longitud, señalando que las hembras T. murphyi en la 
costa de Chile madurarían alrededor de los $36 \mathrm{~cm} \mathrm{LH.} \mathrm{Este}$ antecedente podría ser evidencia de una fuerte reducción en el parámetro $\mathrm{L}_{50}$. No obstante, la variedad de métodos usados en la estimación y los posibles sesgos asociados al tamaño de los ejemplares, impiden obtener conclusiones definitivas sobre una posible reducción en la longitud de madurez de las hembras de jurel en la costa de Chile como consecuencia de la intensa actividad de pesca.

\section{Agradecimientos}

Los autores agradecen al Gobierno de Chile, quienes financiaron el estudio a través de la Subsecretaría de Pesca. De la misma manera, agradecen al Dr. Fran Saborido-Rey y a los investigadores nacionales que participaron del taller donde se discutieron y definieron los criterios de asignación de las fases de madurez usadas en este estudio: Fernando Balbontín, Paola Gonzales, Alexandre Gretchina, Gustavo Herrera, Alberto Olivares, Ciro Oyarzún y Aquiles Sepúlveda. Especial reconocimiento al esfuerzo del personal del Instituto de Fomento Pesquero (IFOP) que colecta las muestras en terreno. Finalmente, se agradecen los valiosos comentarios y sugerencias realizados sobre el manuscrito por los revisores anónimos y editores.

\section{LiTERATURA CITADA}

Abaunza P, L Gordo, C Karlou-Riga, A Murta, A Eltink, M García Santamaría, C Zimmermann, C Hammer, P Lucio \& S Iversen. 2003. Growth and reproduction of horse mackerel, Trachurus trachurus (carangidae). Reviews in Fish Biology and Fisheries 13: 27-61.

Abramov A \& A Kotlar. 1980. Some biological feature of Peruvian jack mackerel Trachurus symmetricus murphyi. Journal of Ichthyology 20(1): 25-31.

Alegría V, J Oliva, H Robotham, H Miranda, G Böhm, L Caballero, D Boré, U Parker, V Baros, S Peña, G Claramunt, G Herrera, C Padilla, P Pizarro, M Medina, H Arancibia, M Araya, L Cubillos, R Gili, D Garland, H Hidalgo \& M Oliva. 1995. Estudio biológico pesquero sobre el recurso jurel en la zona norte (Regiones I y II). Informes Técnicos FIP-IT/93-17: 1-221.

Andrianov DP. 1985. Study on the reproduction of Peruvian scad, Trachurus murphyi (Carangidae), of the Peruvian shelf. Journal of Ichthyology 25: 32-40.

Aracena O, R Alarcón, S Collado, I Lepe \& D Arriagada. 1998. Aspectos reproductivos del jurel (Trachurus symmetricus murphyi) de la pesquería de cerco de la octava región, Chile 1994-1995. En: Arcos D (ed). Biología y ecología del jurel en aguas chilenas, pp. 101-114. Instituto de Investigación Pesquera, Talcahuano.
Arancibia H \& L Cubillos. 1993. Análisis de la pesquería industrial de cerco, dinámica poblacional y situación del recurso jurel, Trachurus symmetricus murphyi. Documento Técnico 2: 1-23, Instituto Investigación Pesquera (IIP), Talcahuano.

Araya M \& R Pepe-Victoriano. 2010. La constancia de la razón entre la longitud de madurez y la longitud asintótica en peces de la costa de Chile: Un meta-análisis. Revista de Biología Marina y Oceanografía 45: 371-377.

Brown-Peterson N, D Wyanski, F Saborido-Rey, B Macewicz \& S Lowerre-Barbierie. 2011. A standardized terminology for describing reproductive development in fishes. Marine and Coastal Fisheries: Dynamics, Management, and Ecosystem Science 3: 52-70.

Conover DO \& S Munch. 2002. Sustaining fisheries yields over evolutionary time scales. Science 297: 94-96.

Cubillos L \& C Alarcón. 2010. Estimación de la talla media de madurez sexual en Trachurus murphyi mediante parámetros del consumo relativo de oxígeno. Latin American Journal of Aquatic Research 38(2): 178-187.

Fenberg PB \& K Roy. 2008. Ecological and evolutionary consequences of size-selective harvesting: how much do we know? Molecular Ecology 17: 209-220.

Grechina A, S Núñez \& D Arcos. 1998. Biología reproductiva del jurel (Trachurus symmetricus murphyi) en el Pacífico sur. En: Arcos D (ed). Biología y ecología del jurel en aguas chilenas, pp. 77-79. Instituto de Investigación Pesquera, Talcahuano.

Holden M \& D Raitt. 1975. Manual de ciencia pesquera. Parte 2: Métodos para investigar los recursos y su aplicación. Documento Técnico de Pesca 115, Rev. 1: 1-211.

Hunter J \& B Macewicz. 2003. Improving the accuracy and precision of reproductive information used in fisheries. In: Kjesbu OS, JR Hunter \& PR Witthames (eds). Report of the working group on modern approaches to assess maturity and fecundity of warm- and cold-water fish and squids, pp. 57-68. Institute of Marine Research, Bergen.

Kaiser CE. 1973. Gonadal maturation and fecundity of horse mackerel Trachurus murphyi (Nichols) off coast of Chile. Transactions of the American Fisheries Society 102: 101108.

Kochkin CE. 1994. Age determination and estimate of growth rate for Peruvian jack mackerel (Trachurus symmetricus murphyi). Journal of Ichthyology 34(3): 39-50.

Kjesbu O, S Hunter \& P Witthames. 2003. Report of the working group on modern approaches to assess maturity and fecundity of warm- and cold-water fish and squids, Fisken og. Havet 12: 117-124.

López L, C Leyton \& M Graf. 1982. Técnicas de histología y citología, 141 pp. Departamento de Biología Celular y Genética, Facultad de Medicina, Universidad de Chile, Santiago. 
Lowerre-Barbieri S, K Ganias, F Saborido-Rey, H Murua \& J Hunter. 2011a. Reproductive timing in marine fishes: Variability, temporal scales, and methods. Marine and Coastal Fisheries: Dynamics, Management, and Ecosystem Science 3: 71-91.

Lowerre-Barbieri S, M Brown-Peterson, H Murua, J Tomkiewicz, D Wyanski \& F Saborido-Rey. 2011b. Emerging issues and methodological advances in fisheries reproductive biology. Marine and Coastal Fisheries: Dynamics, Management, and Ecosystem Science 3: 32-51.

Lucio P, H Murua \& M Santurtún. 2000. Growth and reproduction of hake (Merluccius merluccius) in the Bay of Biscay during the period 1996-1997. Ozeanografika 3: 325-354.

Marshall A, D Frey, D Huppert, E Knaggs, J Macmillan \& D Stanffer. 1980. Biology and economics of fishery for Jack mackerel in the northeast Pacific. NOAA Technical Memorandum NMFS-SWFC-4: 1-85.

Matsumura S, R Arlinghaus \& U Dieckmann. 2011. Assessing evolutionary consequences of size-selective recreational fishing on multiple life-history traits, with an application to northern pike (Esox lucius). Evolutionary Ecology 25: 711-735.

Murua H \& F Saborido-Rey. 2003. Female reproductive strategies of marine fish species of the North Atlantic. Journal of Northwest Atlantic Fisheries Science 33: 23-31.

Murua H, G Kraus, F Saborido-Rey, A Thorsen, P Witthames \& S Junquera. 2003. Procedures to estimate fecundity of wild collected marine fish in relation to fish reproductive strategy. Journal of Northwest Atlantic Fishery Science 33: $33-54$
Nikolsky GV. 1963. The ecology of fishes, 352 pp. Academic Press, London.

Oyarzún C, J Chong \& M Malagueño. 1998. Fenología reproductiva en el jurel, Trachurus symmetricus (Ayres, 1855) (Perciformes, Carangidae) en el área de TalcahuanoChile: 1982-1984. En: Arcos D (ed). Biología y ecología del jurel en aguas chilenas, pp. 67-75. Instituto de Investigación Pesquera, Talcahuano.

Pauly D. 1994. On the sex of fish and the gender of scientists: essays in fisheries science. Fish and Fisheries Series, 264 pp, Chapman and Hall, London.

Piñeiro C \& M Saínza. 2003. Age estimation, growth and maturity of the European hake (Merluccius merluccius (Linnaeus, 1758)) from Iberian Atlantic waters. ICES Journal of Marine Science 60: 1086-1102.

Rijnsdorp A \& B Ibelings. 1989. Sexual dimorphism in the energetic of reproduction and growth of North Sea plaice, Pleuronectes platessa L. Journal of Fish Biology 35: 401415.

Roa R, B Ernst \& F Tapia. 1999. Estimation of size at sexual maturity: an evaluation of analytical and resampling procedures. Fishery Bulletin 97: 570-580.

Rochet MJ. 2009. Effects of fishing on the population. In: Jakobsen T, MJ Fogarty, BA Megrey \& E Moksness (eds). Fish reproductive biology, pp. 172-206. Wiley-Blackwell Scientific Publications, Chichester.

Tomkiewicz J, L Tybjerg \& A Jesperson. 2003. Micro and macroscopic characteristics to stage gonadal maturation of female Baltic cod. Journal of Fish Biology 62: 253-275.

Recibido el 9 de enero 2013 y aceptado el 29 de noviembre de 2013

Editor Asociado: Mauricio Landaeta D. 